\title{
Induction of cell proliferation, clonogenicity and cell accumulation in $S$ phase as a consequence of human UBE2Q1 overexpression
}

\author{
MOHAMMAD ALI FAHMIDEHKAR ${ }^{1}$, SAYED MOHAMMAD SHAFIEE ${ }^{1}$, \\ EBRAHIM EFTEKHAR ${ }^{2}$, LALEH MAHBUDI ${ }^{1}$ and ATEFEH SEGHATOLESLAM ${ }^{1,3}$ \\ ${ }^{1}$ Department of Biochemistry, School of Medicine, Shiraz University of Medical Sciences, Shiraz 71348-45794;
${ }^{2}$ Food and Cosmetic Health Research Center, Hormozgan University of Medical Sciences, Bandar Abbas 79158-73665;
${ }^{3}$ Histomorphometry and Stereology Research Center, Shiraz University of Medical Sciences, Shiraz 71439-14693, Iran
}

Received April 23, 2015; Accepted June 17, 2016

DOI: $10.3892 / 01.2016 .4860$

\begin{abstract}
Ubiquitination is an important cellular mechanism with a pivotal role in the degradation of abnormal or short-lived proteins and the regulation of cell cycle and cell growth. The ubiquitin-proteasome pathway is altered in multiple types of human malignancies, including colorectal cancer (CRC). The alteration in the expression of the novel human gene ubiquitin-conjugating enzyme E2 Q1 (UBE2Q1), as a putative member of the E2 ubiquitin-conjugating enzyme family, has been reported in several malignancies, including carcinoma of the breast, hepatocellular and colorectal cancer, and pediatric acute lymphoblastic leukemia. In the present study, the effect of UBE2Q1 overexpression on cell growth, clonogenicity, motility and cell cycle was investigated in a CRC cell line. The UBE2Q1 gene was cloned in the pCMV6-AN-GFP expression vector. A series of stable transfectants of SW1116 cells overexpressing UBE2Q1 protein were established and confirmed by fluorescence microscopy and western blotting. Using these cells, MTT assay was performed to evaluate cell growth and proliferation, while crystal violet staining was used for clonogenicity assay. Cell cycle analysis was also performed to survey the ratio of cells accumulated in different phases of the cell cycle upon transfection. The motility of these cells was also studied using wound healing assay. UBE2Q1 transfectants exhibited a faster growth in cell culture, increased colony formation capacity and enhanced motility compared with control non-transfected cells and cells transfected with empty vector (mock-transfected cells). UBE2Q1 overexpression also resulted in a significant decrease in the number of cells accumulated in the G0/G1 phase of the cell cycle. The present findings suggest that UBE2Q1 may
\end{abstract}

Correspondence to: Professor Atefeh Seghatoleslam, Department of Biochemistry, School of Medicine, Shiraz University of Medical Sciences, 5th Floor, Building No. 3, Zand Street, Shiraz 71348-45794, Iran

E-mail: seghatolea@sums.ac.ir; seghatoleslama@yahoo.com

Key words: UBE2Q1, transfection, proliferation, clonogenicity, cell cycle, motility, colorectal cancer cells function as an oncogene that induces proliferation of cancer cells, and could be a novel diagnostic tool and a potential therapeutic target for CRC.

\section{Introduction}

Ubiquitination is a critical mechanism for selective protein degradation in eukaryotic cells, playing a pivotal role in protein homeostasis (1). Components of the ubiquitin-proteasome system (UPS) are under the control of three types of enzymes: Ubiquitin-activating enzymes (E1), ubiquitin-conjugating enzymes (E2) and ubiquitin-protein ligases (E3), which recognize and transfer ubiquitin, a 76-amino acids molecule, to the specific target proteins (1-3). Ubiquitin-dependent proteolysis is involved in various cellular biological processes, including control of cell proliferation, transduction, transcription, development, apoptosis and cell cycle (4). This pathway is altered in various types of human cancer, including colorectal cancer (CRC).

$\mathrm{CRC}$ is the third most common type of cancer in males and the second in females throughout the world $(5,6)$. In spite of improvements in diagnosis and adjuvant therapy of CRC (7-9), the mortality rate for CRC is still $40 \%$ (10). Therefore, a critical study for uncovering the biological mechanisms underlying the progression of CRC could lead to more efficient treatment strategies (11).

Ubiquitin-conjugating enzyme E2 Q1 (UBE2Q1), as a novel human protein, is one of the components of the ubiquitin-proteasome pathway, and belongs to the E2 family of enzymes. Its gene is located on chromosome 1 and distributed over $10.454 \mathrm{~kb}$ of genomic DNA. The full-length UBE2Q1 complementary DNA has 13 exons, 3,223 bp and an open reading frame of 1,269 bp encoding a protein of 422 amino acids with a molecular weight of $46.127 \mathrm{kDa}$ [UniProt (http://www.uniprot. org) database number Q7Z7E8-1] (12). Our previous studies revealed that the UBE2Q1 protein was upregulated in human breast and colorectal tumors $(12,13)$, while its expression level was extremely low in numerous normal breast and colorectal tissues. Its upregulation was also demonstrated in hepatocellular carcinoma (9). One of the homologs of the UBE2Q1 gene, UBE2Q2 [also called LOC92912, and renamed by the Human Genome Organization Gene Nomenclature Committee (http:// 
www.genenames.org) as UBE2Q2], is also highly expressed in several human cancers, including head and neck squamous cell carcinoma (HNSCC) (14), breast cancer (3), acute lymphoblastic leukemia (ALL) (15) and CRC (16). The protein products of these two human genes have a ubiquitin conjugate/RWD-like conserved domain at their N-terminal and a UBE2 domain at their C-terminal $(12,14)$.

SW1116 is a colon cancer cell line with a low level of UBE2Q1 messenger (m)RNA and protein expression (13). In the present study, a series of stable UBE2Q1-overexpressing SW1116 cells were established, and the effects of UBE2Q1 overexpression on the proliferation, motility, clonogenicity and cell cycle of these cells were examined.

\section{Materials and methods}

Cell culture, expression vectors and transfection. The human CRC cell line SW1116 was obtained from the National Cell Bank of Iran (Pasteur Institute of Iran, Tehran, Iran). Proliferating SW1116 cells were routinely cultured in RPMI 1640 medium (Gibco; Thermo Fisher Scientific, Inc., Waltham, MA, USA) supplemented with $10 \%(\mathrm{v} / \mathrm{v})$ fetal bovine serum (FBS; Gibco; Thermo Fisher Scientific, Inc.), 100 U/ml penicillin and $100 \mu \mathrm{g} / \mathrm{ml}$ streptomycin at $37^{\circ} \mathrm{C}$ in an incubator with a humidified atmosphere of $5 \% \mathrm{CO}_{2}$. Transfection was performed using Lipofectamine ${ }^{\mathrm{TM}} 2000$ according to the manufacturer's protocol (Invitrogen; Thermo Fisher Scientific, Inc.). A total of $5 \times 10^{4}$ cells were seeded into 24 -well plates in $500 \mu 1$ RPMI 1640 medium containing 10\% FBS without antibiotics, and transferred to the incubator until $\sim 70 \%$ confluency. The pCMV6-AN-GFP-UBE2Q1 expression vector was used to establish cells overexpressing UBE2Q1 tagged with green fluorescent protein (GFP), while the pCMV6-AN-GFP expression vector was used to establish empty vector-transfected cells (OriGene Technologies, Inc., Rockville, MD, USA), which served as a negative control. Non-transfected cells were also used in the present study as an additional negative control. To obtain stable transfectants, $600 \mu \mathrm{g} / \mathrm{ml}$ geneticin (G418; Roche Applied Science, Rotkreuz, Switzerland) was added to the growth medium, starting $48 \mathrm{~h}$ after transfection. The efficiency of transfection was assessed by fluorescence microscopy (Helmut Hund GmbH, Wetzlar, Germany) of GFP-tagged UBE2Q1 protein, and confirmed by western blot analysis. The selection medium was replaced every 3 days, and individual clones (7-8) were isolated and propagated 21-28 days post-transfection. These clones were isolated as stable transfectants.

Western blot analysis of UBE2Q1 protein. pCMV6-AN-GFP-UBE2Q1-transfected, pCMV6-AN-GFP -transfected and non-transfected cells were washed three times with ice-cold phosphate-buffered saline (PBS), scraped separately, and lysed in cell lysis buffer containing $150 \mathrm{mM}$ sodium chloride, $1.0 \%$ (v/v) NP-40 and $50 \mathrm{mM}$ Tris ( $\mathrm{pH} 8.0$ ), which was supplemented with Protease Inhibitor Cocktail (Sigma-Aldrich, St. Louis, MO, USA). The cell lysates were maintained in constant agitation for $30 \mathrm{~min}$ at $4{ }^{\circ} \mathrm{C}$, and finally cleared by sonication and centrifugation. Protein concentrations were determined using Bradford protein assay. Equal amounts of total protein $(30 \mu \mathrm{g})$ were subjected to $12.5 \%$ sodium dodecyl sulfate-polyacrylamide gel electrophoresis (Mini-PROTEAN Tetra Cell; Bio-Rad Laboratories, Inc., Hercules, CA, USA), and then transferred to nitrocellulose membranes (EMD Millipore, Billerica, MA, USA) at $25 \mathrm{~V}$ for $18 \mathrm{~h}$ in the cold room using the Mini Trans-Blot Cell (Bio-Rad Laboratories, Inc.). The membranes were blocked with 5\% non-fat dry milk for $1 \mathrm{~h}$ at room temperature (RT). Immunoblotting was performed with a homemade polyclonal rabbit primary antibody against UBE2Q1 $(1: 2,000)(12)$ and with a primary antibody against $\beta$-actin (Abcam, Cambridge, MA, USA; $1: 1,000$; cat no. 1801) at $4^{\circ} \mathrm{C}$ overnight. After three times washing with PBS-Tween 20 (PBS-T), the membranes were incubated with horseradish peroxidase (HRP)-conjugated secondary antibodies (goat anti-rabbit HRP-conjugated immunoglobulin G; Abcam; 1:2,500; cat no. 6721) at a concentration of $1 \mu \mathrm{g} / \mathrm{ml}$ in $2 \%$ (w/v) bovine serum albumin (Sigma-Aldrich) in PBS-T for $1 \mathrm{~h}$ at RT. Immunoreactive bands were detected with an enhanced chemiluminescence substrate (Chemiluminescent HRP detection kit; Bio-Rad Laboratories, Inc.) and visualized on X-ray films (Agfa HealthCare; Mortsel, Belgium). The expression of $\beta$-actin was used to normalize the amounts of protein loaded.

In vitro proliferation assay. 3-(4,5-dimethylthiazol-2-yl)2,5-diphenyltetrazolium bromide (MTT; Sigma-Aldrich) assay was used to evaluate the effect of UBE2Q1 on cell proliferation capacity. The cells were seeded at a density of $2 \times 10^{3}$ cells/well of a 96 -well cell culture plates (total volume of $200 \mu \mathrm{l} / \mathrm{well}$ ) in RPMI 1640 containing 10\% FBS, and incubated at $37^{\circ} \mathrm{C}$ in a $5 \% \mathrm{CO}_{2}$ incubator. Transfected cells were also supplemented with $2 \mu \mathrm{g} / \mathrm{ml} \mathrm{G} 418$. To measure the cell proliferation after 2, 4 and 6 days of culture, $20 \mu \mathrm{l}$ MTT $(5 \mathrm{mg} / \mathrm{ml})$ solution was added to each well, and the cells were incubated for $4 \mathrm{~h}$ at $37^{\circ} \mathrm{C}, 5 \% \mathrm{CO}_{2}$ and saturated humidity. The medium was then discarded, and the cells were mixed with $100 \mu 1 /$ well dimethyl sulfoxide. The plates were agitated for $15 \mathrm{~min}$ to completely dissolve the formazan crystals, and the absorption values were measured at $570 \mathrm{~nm}$ using a microplate reader (Stat $\mathrm{Fax}^{\circledR}$; Awareness Technology, Inc., Palm City, FL, USA). Six wells were used for each group of cells, including pCMV6-AN-GFP-UBE2Q1-transfected cells, pCMV6-AN-GFP-transfected cells and non-transfected cells. Each experiment was performed three times independently.

Flow cytometry for cell cycle analysis. Cells (transfected and non-transfected) growing in logarithmic phase were washed with PBS, harvested by trypsin/ethylenediaminetetraacetic acid and washed twice with PBS. Then, $10^{6}$ cells were resuspended with $0.5 \mathrm{ml}$ cold PBS and fixed by $70 \%$ (v/v) ice-cold ethanol overnight at $4^{\circ} \mathrm{C}$. The cells were then washed with PBS three times, resuspended in $1 \mathrm{ml}$ propidium iodide (PI) staining solution containing $1 \%$ Triton-X 100, $10 \mu \mathrm{g} / \mathrm{ml}$ PI (Sigma-Aldrich) and $100 \mu \mathrm{g} / \mathrm{ml}$ RNase A in PBS, and kept in the dark at RT for $30 \mathrm{~min}$. The DNA content of the cells was then evaluated by flow cytometry (FACSCalibur, BD Biosciences, Franklin Lakes, NJ, USA), and the data were analyzed using FlowJo version 7.6.1 software (FlowJo, LLC, Ashland, OR, USA).

Colony formation assay. Three groups of cells were seeded for evaluation of colony formation in 6-well plates at a density of 
300 cells/well. After 10 days, the cells were washed with PBS and then stained with $0.05 \%$ crystal violet solution for $1 \mathrm{~h}$. The number of colonies containing $>50$ cells were counted under a light microscope. Photographs were taken using a Nikon COOLPIX 775 camera. The experiments were performed three times independently.

Wound healing assay. The measurement of wound healing (cell motility) activity was performed using 6-well plates. The cells were cultured until confluence, and then serum starved for $24 \mathrm{~h}$. A scratch wound was created in the cell monolayer with a $10-\mu 1$ pipette tip. The cells were then washed twice with PBS to remove the floating cells, and cultured in fresh medium. The progress of cell movement into the wound area was observed at different time intervals $(6,12,18,24,30$ and $36 \mathrm{~h})$, and the representative fields were photographed using a digital camera attached to a microscope (Olympus Corporation, Tokyo, Japan). The percentage of wound closure was measured with OLYSIA BioReport imaging software (Informer Technologies, Inc., Madrid, Spain). Each assay was performed in triplicate, and one representative experiment is shown.

Statistical analysis. All statistical analyses were performed using SPSS 16.0 statistical software (SPSS, Inc., Chicago, IL, USA). Mann-Whitney U test was used to compare data between two groups. $\mathrm{P}<0.05$ was considered to indicate a statistical significant difference. Data are presented as the mean \pm standard deviation.

\section{Results}

Establishment of stable UBE2Q1-overexpressing SW1116 cells. Stable UBE2Q1-overexpressing SW1116 cells and control cells transfected with empty vector were established and maintained in RPMI 1640 containing $600 \mu \mathrm{g} / \mathrm{ml} \mathrm{G} 418$. The transfection efficiency was confirmed primarily by observation of GFP expression under a fluorescence microscope. To confirm the overexpression of UBE2Q1 protein, total cellular protein was extracted using cell lysis buffer from SW1116 cells transfected with the pCMV6-AN-GFP-UBE2Q1 vector, and compared with the empty vector-transfected and non-transfected cells (used as controls). The expression pattern of UBE2Q1 protein was verified by western blot analysis using an anti-UBE2Q1 antibody. UBE2Q1 protein overexpression was confirmed in SW1116 cells transfected with pCMV6-AN-GFP-UBE2Q1 vector at the size expected $69.127 \mathrm{kDa}$ for GFP-UBE2Q1, which corresponds to $46.127 \mathrm{kDa}$ for UBE2Q1 plus $23 \mathrm{kDa}$ for GFP) (Fig. 1).

Effect of UBE2Q1 overexpression on SW1116 cell growth and proliferation. An equal number of SW1116 cells stably transfected with pCMV6-AN-GFP-UBE2Q1 or pCMV6-AN-GFP vector and non-transfected cells were seeded and utilized to evaluate the effect of UBE2Q1 overexpression on cell proliferation. The cell growth curves (Fig. 2) revealed that 4 and 6 days after seeding, the optical density values of the UBE2Q1-transfected cells were significantly higher than those of the cells in the empty vector-transfected and non-transfected groups $(\mathrm{P}<0.05)$. No significant difference was observed between the empty vector group and the non-transfected group. These

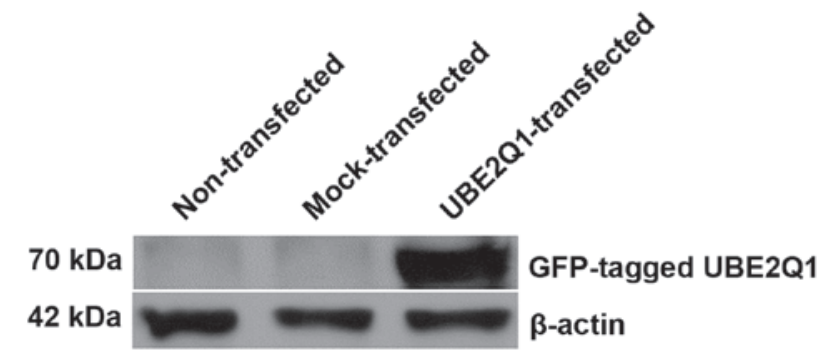

Figure 1. Western blot analysis of UBE2Q1 protein. The right lane shows a $\sim 70 \mathrm{kDa}$ protein band representing green fluorescent protein-tagged UBE2Q1 protein. No intense band was observed for mock-transfected or non-transfected cells. Anti- $\beta$-actin antibody was used as an internal control. UBE2Q1, ubiquitin-conjugating enzyme E2 Q1; GFP, green fluorescent protein.

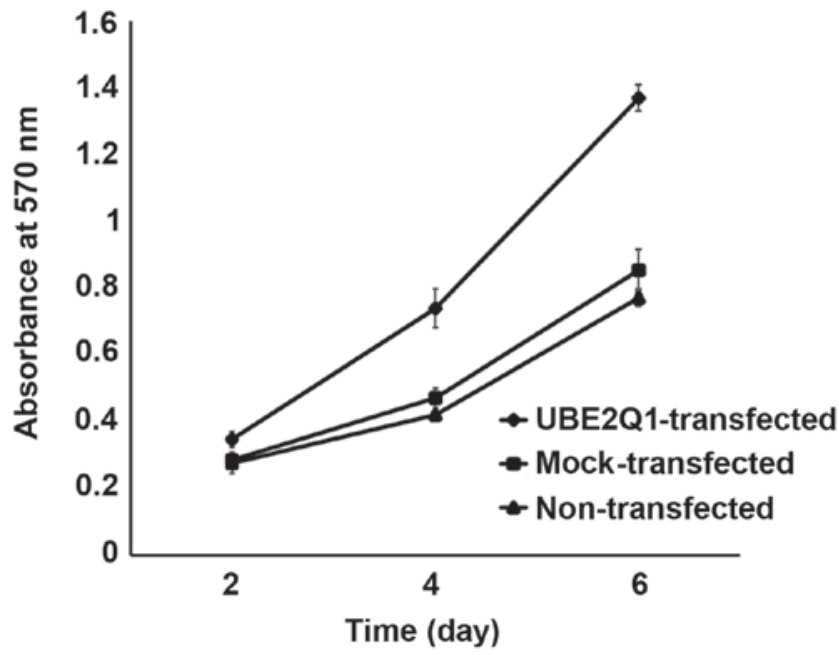

Figure 2. Effect of UBE2Q1 overexpression on SW1116 cell proliferation. The results obtained from 3-(4,5-dimethylthiazol-2-yl)-2,5-diphenyltetrazolium bromide assay revealed a significant increase in cell proliferation on days 4 and 6 in the cells overexpressing UBE2Q1 protein compared with the control mock-transfected and non-transfected cells. Values are represented as the mean \pm standard deviation of two independent experiments performed in triplicate. UBE2Q1, ubiquitin-conjugating enzyme E2 Q1.

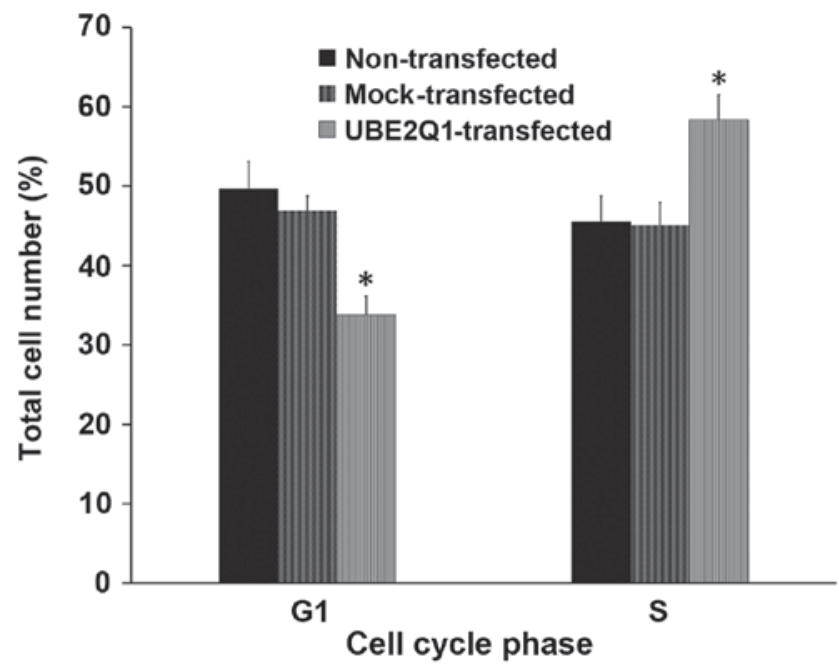

Figure 3. Cell cycle analysis of UBE2Q1-overexpressing cells demonstrated that UBE2Q1 protein induces cell accumulation in S phase and decreases the number of cells accumulated in G1 phase compared with mock-transfected and non-transfected cells (" $\mathrm{P}<0.05$ vs. controls). UBE2Q1, ubiquitin-conjugating enzyme E2 Q1. 
A

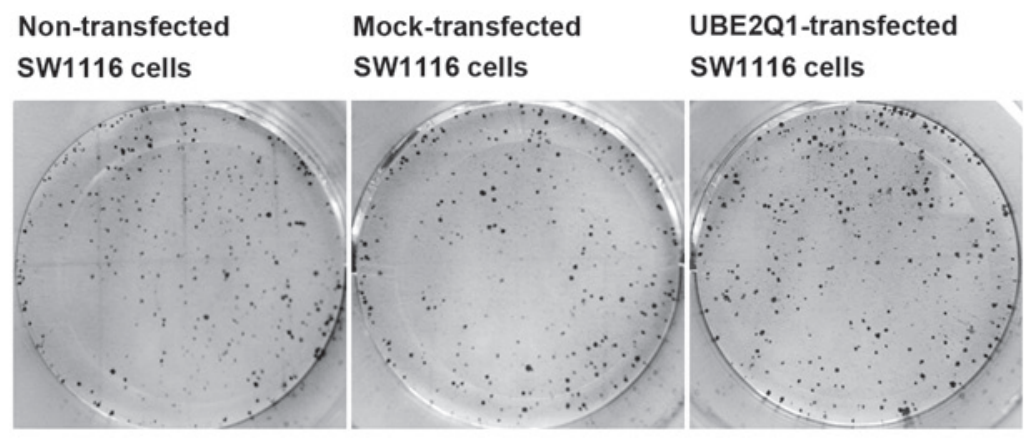

B

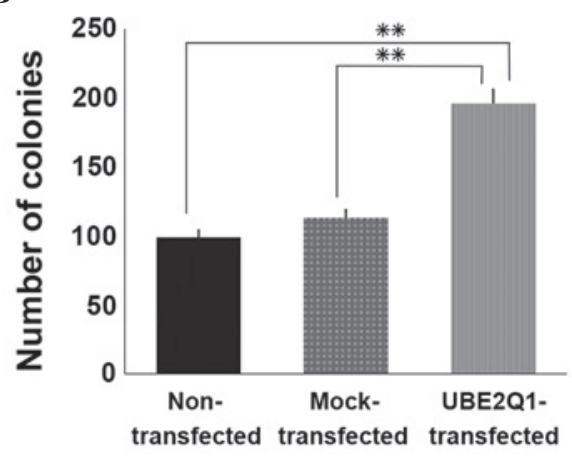

Figure 4. Effect of UBE2Q1 overexpression on colony formation in the colorectal cancer cell line SW1116. (A) Ten days after culturing, the cells were stained with crystal violet and the plates were photographed. (B) The colony numbers in the cells transfected with UBE2Q1 were significantly higher than those in the non-transfected and mock-transfected cells. ${ }^{* *} \mathrm{P}<0.05$ vs. controls. UBE2Q1, ubiquitin-conjugating enzyme E2 Q1.
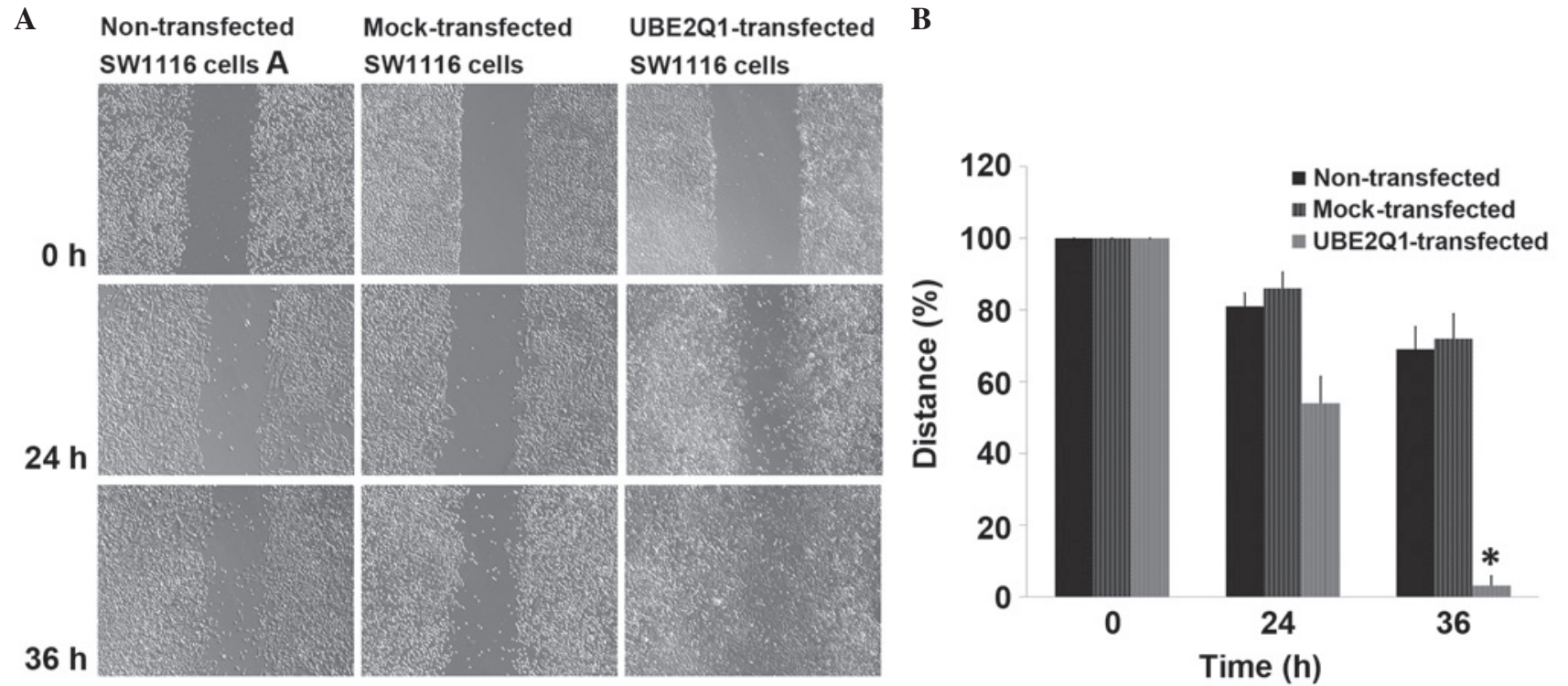

Figure 5. Effect of UBE2Q1 overexpression on the wound healing activity of the colorectal cancer cell line SW1116. (A) At 0, 24 and $36 \mathrm{~h}$ after scratching, the migration of the cells into the scratched area was photographed. Magnification, x10 (B) The distances between the edges of the cells that had migrated from each side of the wound were measured and compared with the initial distances, at $0 \mathrm{~h}$ after scratching, which was considered as $100 \%$. The distances are presented as percentages and shown as the mean \pm standard deviation $(\mathrm{n}=5),{ }^{*} \mathrm{P}<0.01$. UBE2Q1, ubiquitin-conjugating enzyme E2 Q1.

results suggest that the overexpression of UBE2Q1 protein could increase the growth of the CRC cell line, SW1116, in vitro.

Effect of UBE2Q1 overexpression on SW1116 cell cycle. Flow cytometry was used for cell cycle analysis of SW1116 cells transfected with pCMV6-AN-GFP-UBE2Q1 vector, SW1116 cells transfected with pCMV6-AN-GFP empty vector and non-transfected SW1116 cells. The results demonstrated that the percentage of cells in the $\mathrm{G} 0 / \mathrm{G} 1$ phase in relation to the total cell counts in the UBE2Q1-overexpressing cells was significantly low $(33.80 \pm 2.33 \%)$ compared with the empty vector-transfected cells $(46.90 \pm 1.87 \%)$ and the non-transfected cells $(49.70 \pm 3.39 \%)$ $(\mathrm{P}<0.05)$. Similarly, the percentage of cells in the $\mathrm{S}$ phase in relation to the total cell counts in the UBE2Q1-overexpressing cells was significantly high $(58.40 \pm 3.09 \%)$ compared with the empty vector-transfected $(45.10 \pm 2.83 \%)$ and non-transfected cells $(45.50 \pm 3.28 \%)(\mathrm{P}<0.05)$ (Fig. 3). The distribution of the cells in the different phases of the cell cycle exhibited a similar pattern for the cells transfected with empty vector and the non-transfected cells $(\mathrm{P}>0.05)$. These data suggest that a high level of UBE2Q1 expression may promote cell proliferation by facilitating cell cycle progression in SW1116 cells.

Effect of UBE2Q1 on SW1116 colony formation. The effect of UBE2Q1 overexpression on colony formation was evaluated by colony formation assay. As shown in Fig. 4, SW1116 cells overexpressing UBE2Q1 protein displayed a significant increase in colony number and colony size compared with pCMV6-AN-GFP-transfected and non-transfected cells. Therefore, the results suggest that overexpression of UBE2Q1 induces cell growth and colony formation.

Effect of UBE2Q1 overexpression on SW1116 motility and wound healing. The effects of UBE2Q1 overexpression on cell motility activity were investigated using wound healing assay in different time intervals of 24 and $36 \mathrm{~h}$ (Fig. 5A). At $36 \mathrm{~h}$ after scratching, the distances between the boundaries of the wound region were measured and compared with the initial distances 
(0 h after scratching), which were considered as $100.0 \%$. The distances were $3.1 \pm 2.7,72.0 \pm 6.9$ and $69.0 \pm 6.3 \%$ for UBE2Q1-overexpressing cells, empty vector-transfected cells and non-transfected cells, respectively (Fig. 5B). These results confirmed that the migration capacity of SW1116 cells in the UBE2Q1-overexpressing group was significantly higher at $36 \mathrm{~h}$ compared with the negative control groups. These differences were also obvious after $24 \mathrm{~h}$ of scratching. No significant difference was observed between the two negative controls.

\section{Discussion}

Alteration in the expression of the UPS components has been extensively reported to play key roles in carcinogenesis (17). Several E2 ubiquitin-conjugating enzymes have been demonstrated to be closely involved in cell cycle progression and tumorigenesis (18-30). In our previous studies, UBE2Q1 was observed to be predominantly expressed in the majority of breast and colorectal tumor samples analyzed, whereas it was expressed weakly in normal breast and colorectal tissues $(12,13)$. The alteration in UBE2Q1 mRNA expression was also reported in pediatric ALL (31). The expression pattern of UBE2Q1 in several CRC cell lines was previously studied by the present authors, who demonstrated that UBE2Q1 expresses at the lowest mRNA and protein levels in the SW1116 cell line compared with the other examined cell lines (13).

In the present study, using the above cell line, a series of SW1116 cells overexpressing UBE2Q1 were established for the first time, and the effect of UBE2Q1 upregulation on the behavior and function of these cells was investigated and compared with the negative controls. The overexpression of UBE2Q1 protein was firstly confirmed by fluorescence microscopy and western blot analysis of GFP-tagged UBE2Q1 protein. It was observed that UBE2Q1 overexpression induces SW1116 cell growth and proliferation significantly. The results obtained by flow cytometry revealed that overexpression of UBE2Q1 promotes cell progression through the cell cycle by decreasing the number of cells in the G0/G1 phase of the cell cycle, resulting in cell accumulation in the $\mathrm{S}$ phase. In addition, UBE2Q1-overexpressing cells exhibited enhanced colony formation ability compared with negative control cells. Furthermore, cells overexpressing UBE2Q1 displayed higher motility and migration than the negative control when examined in wound healing assay. Cumulatively, the data presented in the current study confirmed that UBE2Q1 could promote the growth, proliferation, migration and clonogenicity of SW1116 cancer cells, which is in agreement with the role suggested for this gene according to the previous studies $(12,13)$. A recent study has shown the association between upregulation of UBE2Q1 and enhanced cell proliferation and poor prognosis in human hepatocellular carcinoma (9). The authors also knocked down UBE2Q1 in HepG2 and BEL-7404 cells using small interfering RNA, and observed a significant increase in the level of p53 and p21 tumor suppressor proteins (9). In another study by Wan et al, the researchers reported that the inhibition of the p53 signaling pathway could decrease UBE2Q1 expression in apoptotic multiple myeloma cells (32). Further studies using immunoprecipitation and GST pull-down assays showed the binding of UBE2Q1 to p53 protein, both in vivo and in vitro. This study also revealed that overexpression of UBE2Q1 can result in the repression of p53 in MDA-MB468 cells; this may be due to UBE2Q1 mediated ubiquitination and subsequent proteasome degradation of p53 (33). These findings, together with the aforementioned altered expression of UBE2Q1 in cancer, indicate the possibility of the regulation of UBE2Q1 by the p53 signaling pathway and its noticeable participation in cancer development. The occurrence of these phenomena may be mediated by the interacting partners of the UBE2Q1 protein in various cell signaling pathways involved in cellular growth, cell cycle and migration. These results open a landscape towards exploring the interacting partners of this novel protein and identifying its upstream and downstream molecules involved in carcinogenesis. UBE2Q1 has been also observed to be a potential binding partner of carboxyl terminus of heat shock protein 70-interacting partner (34), which participates in protein quality control and stress response (35), and functions as an E3 ligase for p53 (36). Notably, UBE2Q2, a close related homolog of UBE2Q1 that belongs to the class II E2 family of enzymes (30), has been demonstrated to be differentially expressed in several human malignancies, including HNSCC (14), breast cancer (3), pediatric ALL (15) and CRC (16). Its participation in the regulation of the cell cycle and check point control (37), and its involvement in the termination of the spindle assembly and chromosomal instability (38), has additionally been reported. Its interacting partners were also reported in 2006 by the present authors to be actin and actin-related proteins (14).

However, the mechanism underlying UBE2Q1 upregulation and its role in cancer biology and development remains largely unknown. Further investigations are required to clarify its precise role in the pathogenesis of human malignancies, particularly CRC.

In summary, the present findings suggest that UBE2Q1 may be important in the regulation of cell proliferation and/or survival, and could be used as a promising marker for the diagnosis and treatment of cancer.

\section{Acknowledgements}

The present study was based on the $\mathrm{PhD}$ thesis written by $\mathrm{Mr}$. Mohammad Ali Fahmidehkar and was financially supported by Shiraz University of Medical Sciences (Shiraz, Iran; grant no. 92-6617).

\section{References}

1. Shen Z, Jiang X, Zeng C, Zheng S, Luo B, Zeng Y, Ding R, Jiang H, He Q, Guo J and Jie W: High expression of ubiquitin-conjugating enzyme 2C (UBE2C) correlates with nasopharyngeal carcinoma progression. BMC Cancer 13: 192, 2013.

2. Grzmil P, Altmann ME, Adham IM, Engel U, Jarry H, Schweyer S, Wolf S, Mänz J and Engel W: Embryo implantation failure and other reproductive defects in Ube2q1-deficient female mice. Reproduction 145: 45-56, 2013.

3. Nikseresht M, Seghatoleslam A, Monabati A, Talei A, Ghalati FB and Owji AA: Overexpression of the novel human gene, UBE2Q2, in breast cancer. Cancer Genet Cytogenet 197: 101-106, 2010.

4. Hershko A: The ubiquitin system for protein degradation and some of its roles in the control of the cell division cycle. Cell Death Differ 12: 1191-1197, 2005.

5. Zhu D, Zhong Y, Wu H, Ye L, Wang J, Li Y, Wei Y, Ren L, Xu B, $\mathrm{Xu} \mathrm{J}$ and Qin X: Predicting metachronous liver metastasis from colorectal cancer using serum proteomic fingerprinting. J Surg Res 184: 861-866, 2013. 
6. Zhou LH, Hu Q, Sui H, Ci SJ, Wang Y, Liu X, Liu NN, Yin PH, Qin JM and Li Q: Tanshinone II-a inhibits angiogenesis through down regulation of COX-2 in human colorectal cancer. Asian Pac J Cancer Prev 13: 4453-4458, 2012.

7. Van Cutsem E, Borràs JM, Castells A, Ciardiello F, Ducreux M, Haq A, Schmoll HJ and Tabernero J: Improving outcomes in colorectal cancer: Where do we go from here? Eur J Cancer 49: 2476-2485, 2013.

8. Kopetz S, Chang GJ, Overman MJ,Eng C, Sargent DJ,Larson DW, Grothey A, Vauthey JN, Nagorney DM and McWilliams RR: Improved survival in metastatic colorectal cancer is associated with adoption of hepatic resection and improved chemotherapy. $\mathrm{J}$ Clin Oncol 27: 3677-3683, 2009.

9. Chang R, Wei L, Lu Y, Cui X, Lu C, Liu L, Jiang D, Xiong Y, Wang $\mathrm{G}$, Wan $\mathrm{C}$, et al: Upregulated expression of ubiquitinconjugating enzyme E2Q1 (UBE2Q1) is associated with enhanced cell proliferation and poor prognosis in human hapatocellular carcinoma. J Mol Histol 46:45-56, 2015.

10. Chen Z, He X, Jia M, Liu Y, Qu D, Wu D, Wu P, Ni C, Zhang Z, Ye J, et al: $\beta$-catenin overexpression in the nucleus predicts progress disease and unfavourable survival in colorectal cancer: A meta-analysis. PLoS One 8: e63854, 2013.

11. Bai R, Li D, Shi Z, Fang X, Ge W and Zheng S: Clinical significance of Ankyrin repeat domain 12 expression in colorectal cancer. J Exp Clin Cancer Res 32: 35, 2013.

12. Seghatoleslam A, Nikseresht M, Shafiee SM, Monabati A, Namavari MM, Talei A, Safaei A and Owji AA: Expression of the novel human gene, UBE2Q1, in breast tumors. Mol Biol Rep 39: 5135-5141, 2012.

13. Shafiee SM, Seghatoleslam A, Nikseresht M, Hosseini SV, Alizadeh-Naeeni M, Safaei A and Owji AA: UBE2Q1 expression in human colorectal tumors and cell lines. Mol Biol Rep 40: 7045-7051, 2013

14. Seghatoleslam A, Zambrano A, Millon R, Ganguli G, Argentini M, Cromer A, Abecassis J and Wasylyk B: Analysis of a novel human gene, LOC92912, overexpressed in hypopharyngeal tumours. Biochem Biophys Res Commun 339: 422-429, 2006.

15. Seghatoleslam A, Monabati A, Bozorg-Ghalati F, Nikseresht M, Bordbar MR, Rahvar M and Owji AA: Expression of UBE2Q2, a putative member of the ubiquitin-conjugating enzyme family in pediatric acute lymphoblastic leukemia. Arch Iran Med 15: 352-355, 2012

16. Shafiee SM, Seghatoleslam A, Nikseresht M, Hosseini SV, Alizadeh-Naeeni M, Safaei A and Owji AA: Expression status of UBE2Q2 in colorectal primary tumors and cell lines. Iran J Med Sci 39 (2 Suppl): S196-S202, 2014.

17. Zhu S, Yao F, Li WH, Wan JN, Zhang YM, Tang Z, Khan S, Wang CH and Sun SR: PKC?-dependent activation of the ubiquitin proteasome system is responsible for high glucose-induced human breast cancer MCF-7 cell proliferation, migration and invasion. Asian Pac J Cancer Prev 14: 5687-5692, 2013.

18. Burger AM and Seth AK: The ubiquitin-mediated protein degradation pathway in cancer: Therapeutic implications. Eur J Cancer 40: 2217-2229, 2004

19. Voutsadakis IA: Ubiquitin- and ubiquitin-like proteins-conjugating enzymes (E2s) in breast cancer. Mol Biol Rep 40: 2019-2034, 2013

20. Hao Z, Zhang $\mathrm{H}$ and Cowell J: Ubiquitin-conjugating enzyme UBE2C: Molecular biology, role in tumorigenesis, and potential as a biomarker. Tumour Biol 33: 723-730, 2012.

21. Seghatoleslam A and Zambrano A: Effects of overexpression of LOC92912 gene on cell cycle progression. Iran J Med Sci 34: 277-284, 2009.

22. Tang XK, Wang KJ, Tang YK and Chen L: Effects of ubiquitin-conjugating enzyme $2 \mathrm{C}$ on invasion, proliferation and cell cycling of lung cancer cells. Asian Pac J Cancer Prev 15: 3005-3009, 2014.
23. Chen S, Chen Y, Hu C, Jing H, Cao Y and Liu X: Association of clinicopathological features with UbcH10 expression in colorectal cancer. J Cancer Res Clin Oncol 136: 419-426, 2010.

24. Pallante P, Berlingieri MT, Troncone G, Kruhoffer M, Orntoft TF, Viglietto G, Caleo A, Migliaccio I, Decaussin-Petrucci M, Santoro M, et al: $\mathrm{UbcH} 10$ overexpression may represent a marker of anaplastic thyroid carcinomas. Br J Cancer 93: 464-471, 2005.

25. Ieta K, Ojima E, Tanaka F, Nakamura Y, Haraguchi N, Mimori $\mathrm{K}$, Inoue $\mathrm{H}$, Kuwano $\mathrm{H}$ and Mori M: Identification of overexpressed genes in hepatocellular carcinoma, with special reference to ubiquitin-conjugating enzyme E2C gene expression. Int J Cancer 121: 33-38, 2007.

26. Berlingieri MT, Pallante P, Sboner A, Barbareschi M, Bianco M, Ferraro A, Mansueto G, Borbone E, Guerriero E, Troncone G and Fusco A: UbcH10 is overexpressed in malignant breast carcinomas. Eur J Cancer 43: 2729-2735, 2007.

27. Bose MV, Gopisetty G, Selvaluxmy G and Rajkumar T: Dominant negative Ubiquitin-conjugating enzyme E2C sensitizes cervical cancer cells to radiation. Int J Radiat Biol 88: 629-634, 2012.

28. Perrotta I, Bruno L, Maltese L, Russo E, Donato A and Donato G: Immunohistochemical analysis of the ubiquitin-conjugating enzyme UbcH10 in lung cancer: A useful tool for diagnosis and therapy. J Histochem Cytochem 60: 359-365, 2012.

29. Lin J, Raoof DA, Wang Z, Lin MY, Thomas DG, Greenson JK, Giordano TJ, Orringer MB, Chang AC, Beer DG and Lin L: Expression and effect of inhibition of the ubiquitin-conjugating enzyme E2C on esophageal adenocarcinoma. Neoplasia 8: 1062-1071, 2006

30. van Wijk SJ and Timmers HT: The family of ubiquitin-conjugating enzymes (E2s): Deciding between life and death of proteins. FASEB J 24: 981-993, 2010.

31. Seghatoleslam A, Bozorg-Ghalati F, Monabati A, Nikseresht M and Owji AA: UBE2Q1, as a down regulated gene in pediatric acute lymphoblastic leukemia. Int J Mol Cell Med 3: 95-101, 2014.

32. Wan C, Chen J, Hu B, Zou H, Li A, Guo A and Jiang J: Downregulation of UBE2Q1 is associated with neuronal apoptosis in rat brain cortex following traumatic brain injury. J Neurosci Res 92: 1-12, 2014

33. Shafiee SM, Rasti M, Seghatoleslam A, Azimi T and Owji AA: UBE2Q1 in a Human Breast Carcinoma Cell Line: Overexpression and Interaction with p53. Asian Pac J Cancer Prev 16: 3723-7, 2015

34. Grelle G, Kostka S, Otto A, Kersten B, Genser KF, Müller EC, Wälter S, Böddrich A, Stelzl U, Hänig C, et al: Identification of $\mathrm{VCP} / \mathrm{p} 97$, carboxyl terminus of Hsp70-interacting protein (CHIP), and amphiphysin II interaction partners using membrane-based human proteome arrays. Mol Cell Proteomics 5: 234-244, 2006.

35. Min JN, Whaley RA, Sharpless NE, Lockyer P, Portbury AL and Patterson C: CHIP deficiency decreases longevity, with accelerated aging phenotypes accompanied by altered protein quality control. Mol Cell Biol 28: 4018-4025, 2008.

36. Naito AT, Okada S, Minamino T, Iwanaga K, Liu ML, Sumida T, Nomura S, Sahara N, Mizoroki T, Takashima A, et al: Promotion of CHIP-mediated p53 degradation protects the heart from ischemic injury. Circ Res 106: 1692-1702, 2010.

37. Banerjee S, Brooks WS and Crawford DF: Inactivation of the ubiquitin conjugating enzyme UBE2Q2 causes a prophase arrest and enhanced apoptosis in response to microtubule inhibiting agents. Oncogene 26: 6509-6517, 2007.

38. Melner MH, Haas AL, Klein JM, Brash AR, Boeglin WE, Nagdas SK, Winfrey VP and Olson GE: Demonstration of ubiquitin thiolester formation of UBE2Q2 (UBCi), a novel ubiquitin-conjugating enzyme with implantation site-specific expression. Biol Reprod 75: 395-406, 2006. 